\title{
Comparative Analysis of Follicle Stimulating Hormone Beta-Subunit Gene in Mammals
}

\author{
Qin Wang ${ }^{1} \&$ Chengzhong Yang ${ }^{2}$ \\ ${ }^{1}$ Department of Biology, College of Life Sciences, Sichuan Agricultural University, Yaan, PR China \\ ${ }^{2}$ School of Life Sciences, Jinggangshan University, Ji'an, Jiangxi, PR China \\ Correspondence: Chengzhong Yang, School of Life Sciences, Jinggangshan University, Ji'an, Jiangxi Province \\ 343009, PR China. E-mail: chzhongyang@gmail.com
}

Received: April 2, 2013 Accepted: May 2, 2013 Online Published: May 15, 2013

doi:10.5539/jas.v5n6p20 URL: http://dx.doi.org/10.5539/jas.v5n6p20

\begin{abstract}
Follicle stimulating hormone (FSH) is a glycoprotein hormone expressed by gonadotropes in the pituitary gland that regulated the development and function of gonads and is necessary for normal propagation functions in mammals. In this study, FSH $\beta$ revealed a high degree of conservation by contrasted with homologous fragment from serviceable mammals. These results suggested an insight into the characteristics of FSH $\beta$. Using the software of RepeatMasker and Alignment, the content and distribution of SINE/tRNA-Glu and LINE/L1 in pig FSH $\beta$ subunit were detected. These insertion elements did not emerged in other artiodactylous FSH $\beta$. It is probable positive selection during the evolutionary process of pig in artificial selection.
\end{abstract}

Keywords: mammals, evolution, FSH $\beta$, analysis

\section{Introduction}

Follicle-stimulating hormone (FSH), luteinizing hormone (LH), chorionic gonadotropin (CG) and thyroid-stimulating hormone belong to the family of glycoprotein hormones, and the chorionic gonadotropin (CG) has been found only in the placenta of equine species and primates. These hormones are heterodimers consisting of non-covalently linked common $\alpha$ and hormone-specific $\beta$ subunits. FSH is responsible for development and survival of follicular somatic cells, and inducting of ovarian estrogen in women. In males, FSH regulates spermatogenesis by binding to the Sertoli cells (McGee \& Hsueh, 2001; Plant \& Marshall, 2001). Because of its necessary for unique mammalian reproduction function, FSH $\beta$ has possess evolutionarily conserved (Wallis, 2001). The FSH $\beta$ gene is characterized by excess of multiformity with allele frequencies and low genetic variation (Grigorova et al., 2007). Rearrangement sequence study identified a density of polymorphisms of $3 \mathrm{SNPs} / 1 \mathrm{~kb}$ in three human populations (European Estonians, Chinese Han and African Mandenkalu). Majority of these were common polymorphisms located in non-coding regions and were shared by three human populations. Although many FSH models were exist to deliberate the effects of gonadotropins on testicular functionand and testosterone as well as its role in spermatogenesis (Zheng et al., 1998). Sun et al. (2006) showed that FSH has a direct role in causing hypogonadal bone loss. Little attention has been paid to the interspecific evolution of FSH. In this study, we focused on nucleotide variation in order to demonstrate molecular evolution of the analyzed species and to provide useful data for studying the relationship of nucleotide variation in interspecific FSH $\beta$ gene.

\section{Materials and Methods}

In the present research, mRNA sequences of FSH $\beta$ in 20 species and DNA fragments in 15 sequences were downloaded from GenBank. All the sequences were aligned before the phyletic evolution tree was conducted. To avoid redundant sequences, the aligned nucleotide sequences were classified into different haplotypes using DNASP 4.0. The polymorphism site, the number of total mutations (M), the synonymous nucleotide $\operatorname{diversity}[\mathrm{p}(\mathrm{s})]$, number of haplotypes $(\mathrm{h})$, nonsynonymous nucleotide diversity [p(a)], the average number of nucleotide differences (Tajima, 1983) were calculated by DNASP 4.0. The Molecular Evolutionary Genetic Analysis (MEGA version 4.0) package (Tamura et al., 2007) was used to complete deletion of gaps. The Neighbor-Joining (NJ) method through bootstrap analysis (1000 pseudoreplicates) was used to construct two phylogenetic trees using the computer program MEGA. Repeats sequences were analyzed in repeat masker 


\section{(http://repeatmasker.org/).}

\section{Results and Discussion}

Through analysis it was found that the full coded sequences of FSH $\beta$ ranges from 1827 (Ovis aries) to 1984 bp (Ornithorhynchus anatinus). Whereas, FSH $\beta$ identity varied from $100 \%$ for Macaca fascicularis-Macaca mulatta comparisons to $70 \%$ homology between Cervus nippon and Ornithorhynchus anatinus at the nucleotide level; this represents 100 and $65 \%$ similarity at the amino acid level, respectively. Primates had the lowest diversity compared to the average variation among all of the mammals. And the average nucleotide differences and nucleotide diversity are 0.172 and 0.043 , respectively. Hence, the exogenous hormone of related species hormone or homogeneous hormones should be more conducive to captive breeding success.

Moschus berezovskii
Cervus nippon
Bos taurus
Bubalus bubalis
Capra hircus breed Boer
Sus scrofa
Ovis aries
Equus caballus
Homo
Pan troglodytes
Macaca fascicularis
Macaca mulatta
Aotus nancymaae
Mus musculus
Oryctolagus cuniculus
Panthera tigris altaica
Ailuropoda melanoleuca
Trichosurus vulpecula
Monodelphis domestica
Ornithorhynchus anatinus
Moschus berezovskii
Cervus nippon
Bos taurus
Bubalus bubalis
Capra hircus breed Boer
Sus scrofa
Ovis aries
Equus caballus
Homo
Pan troglodytes
Macaca fascicularis
Macaca mulatta
Aotus nancymaae
Mus musculus
Oryctolagus cuniculus
Panthera tigris altaica
Ailuropoda melanoleuca
Trichosurus vulpecula
Monodelphis domestica
Ornithorhynchus anatinus

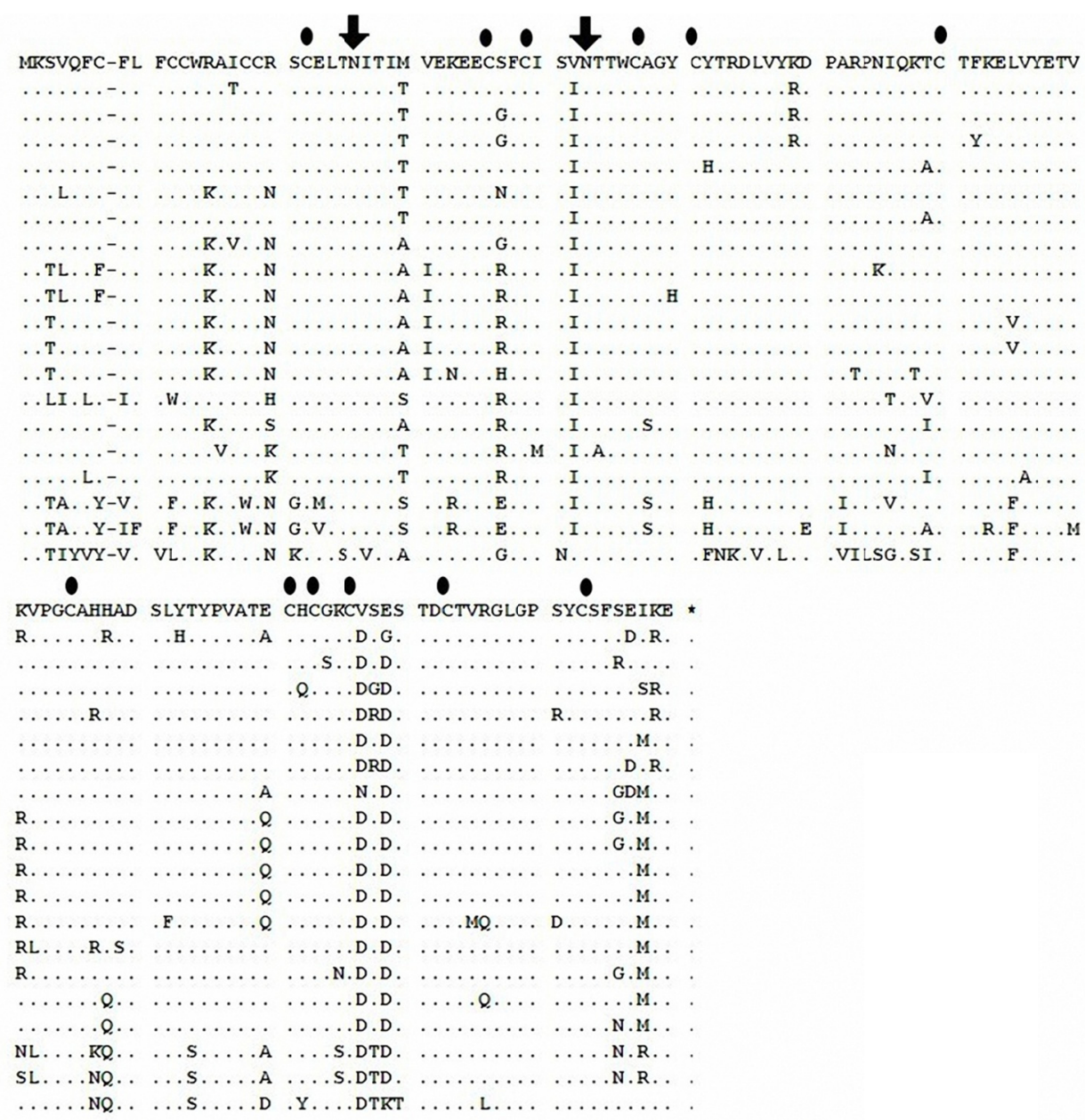

Figure 1. Analyses of amino acid sequence of FSH $\beta$ in mammals

“ $\rightarrow$ " means potential N-linked glycosylation sites, “•”means cysteine residues.

There was a highly conserved among the species in the deduced amino acid sequence. Through these multiple sequence alignments, some common features of FSH $\beta$ in the amino acid sequences were demonstrate. Firstly, within the mature protein region, the same positions of 12 half Cys residues of FSH $\beta$ were the same in the mammals. Six pairs of disulfide bonds of Cys residues are essential not only for the function of heterodimer FSH molecule but also for the correct folding of $\beta$ subunit. Secondly, two putative N-linked glycosylation sites presented at the 25th and 42th amino acid residue in the all FSH $\beta$ subunit (Figure 1); one is located between the first and second Cys residues, while the other is located between the third and fourth Cys residue. Glycosylation of FSH $\beta$ is essential for full bioactivity and protein biosynthesis, especially in protein secretion and folding, safeguard from enzymolysis in recurrence, and the signal conduction pass by receptors-ligands binding (Ulloa-Aguirre et al., 1999). These substantial similarities suggest a common ancestral origin. It was earlier proposed that all members of the glycoprotein gene family (common- $\alpha$, LH $\beta$, FSH $\beta$ and TSH $\beta$ ) evolved from a 
single ancestor through gene duplications. The first duplication produced a-subunit and b-subunit and was followed by a second duplication of the ancestral $\beta$-subunit to yield the LH- $\beta$ subunit gene and the ancestor of the TSH $\beta$ and FSH $\beta$ subunits which eventually gave rise to the genes for these two latter $\beta$-subunits (Li \& Ford, 1998).

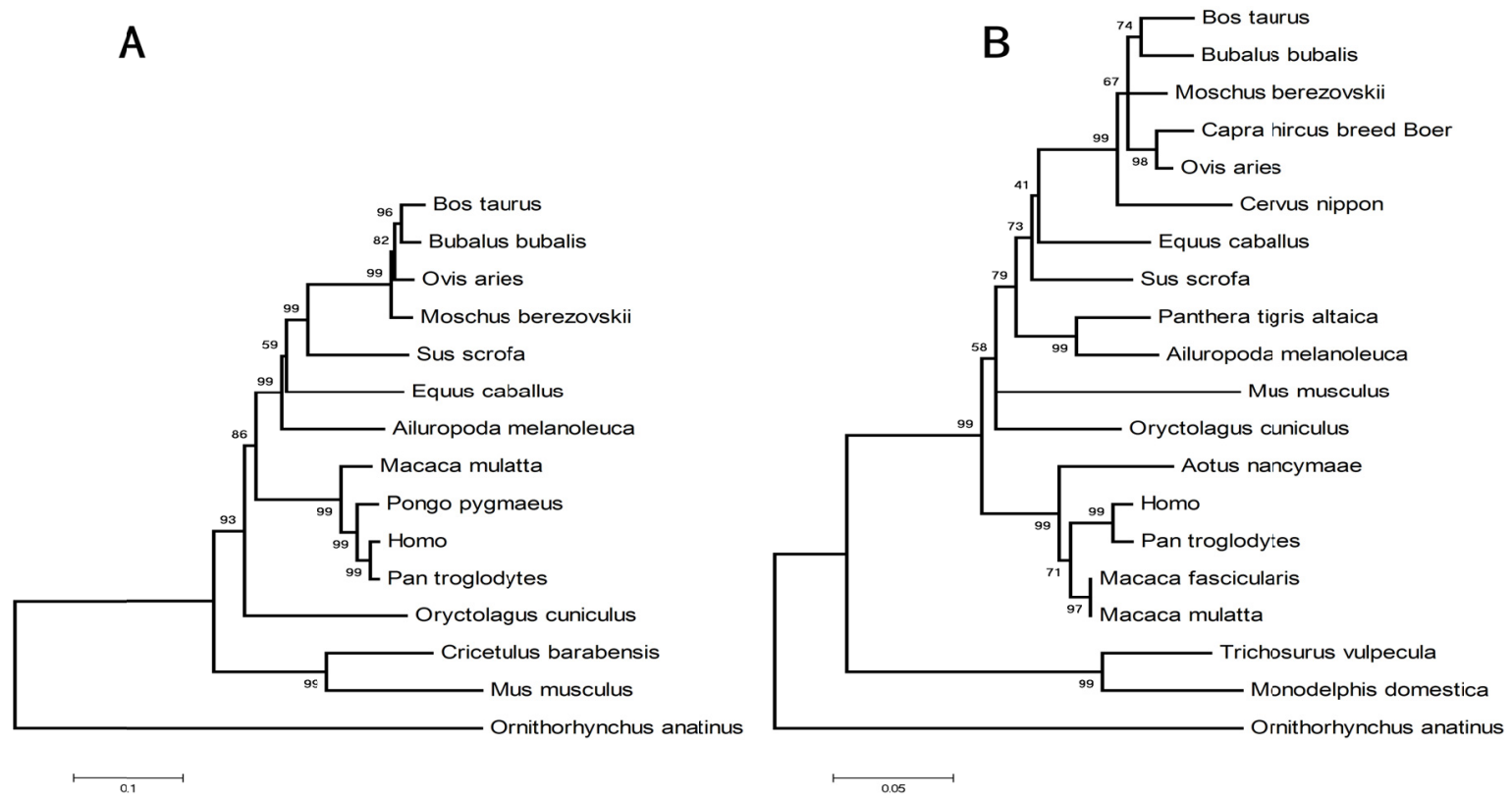

Figure 2. The NJ-tree of mammals based on FSH $\beta$ gene. The node number are Bootstrap values. A was inferred from DNA data and $\mathrm{B}$ from mRNA data

The constructed system evolution tree of FSH $\beta$ of families and species was basically conform to taxology of NCBI except for Sus scrofa, which was clade root of ungulate rather than clustered artiodactyla (Figure 2B). It provided that there was a difference evolution speed of FSH $\beta$ molecule among evolution of amniotic vertebrates (Wallis, 2001).

Using RepeatMasker and sequence alignments of FSH $\beta$ gene identified that porcine FSH $\beta$ subunit exhibits a remarkable different with those of other mammals (Figure 3). The sequence alignments of FSH $\beta$ gene among mammals indicated two microsatellite sites obviously in artiodactyls. However pig FSH $\beta$ contain (CT)n repeats, (AT)n repeats is absent. Whether this additional microsatellite sequence present in artiodactyls FSH $\beta$ is related to litter size and other reproductive traits requires further investigations.

Table 1. The analysis results of repeatmasker (http://repeatmasker.org/) of domestic pig (GenBank: D00621)

\begin{tabular}{|c|c|c|c|c|c|c|c|c|}
\hline \multicolumn{3}{|c|}{ position in query } & \multicolumn{2}{|c|}{ matching repeat } & \multicolumn{4}{|c|}{ position in repeat } \\
\hline begin & end & (left) & repeat & class/family & begin & end & (left) & ID \\
\hline 5997 & 6256 & (3916) & Pre0 SS & SINE/tRNA-Glu & 1 & 260 & (0) & 14 \\
\hline 7054 & 7092 & $(3080)$ & AT_rich & Low_complexity & 1 & 39 & (0) & 15 \\
\hline 7368 & 7439 & (2733) & MIR3 & SINE/MIR & (55) & 153 & 82 & 16 \\
\hline 8055 & 8075 & (2097) & AT rich & Low_complexity & 1 & 21 & (0) & 17 \\
\hline 8817 & 8990 & $(1182)$ & L1-2 SSc & LINE/L1 & (1) & 6015 & 5843 & 18 \\
\hline 9117 & 9223 & (949) & L2b & LINE/L2 & 3246 & 3405 & (21) & 19 \\
\hline 9885 & 9917 & $(255)$ & AT_rich & Low_complexity & 1 & 33 & (0) & 20 \\
\hline 10031 & 10172 & (0) & PRE1 SS & SINE/tRNA-Glu & 1 & 142 & (116) & 21 \\
\hline
\end{tabular}




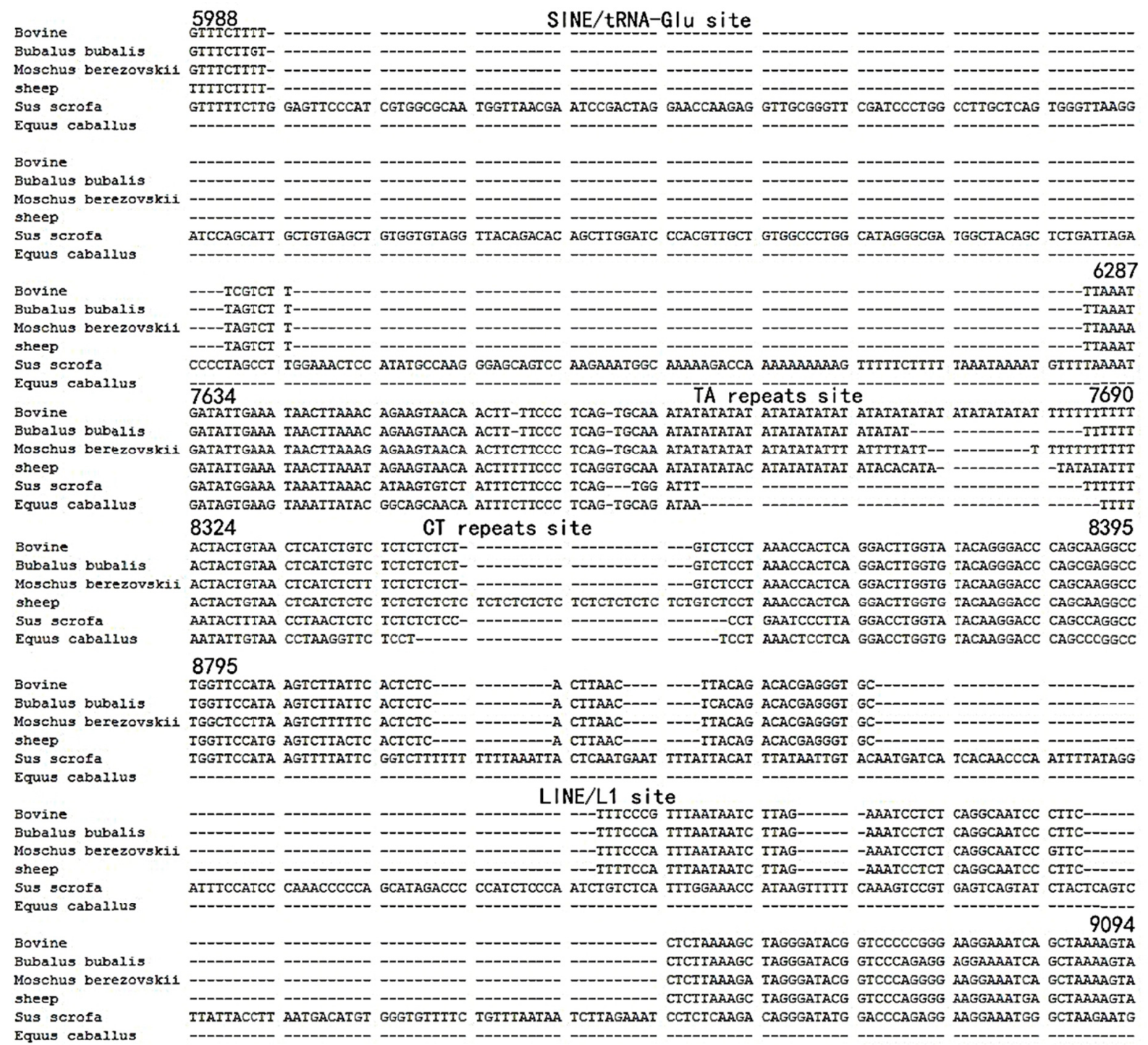

Figure 3. Alignment of FSH $\beta$ gene in Artiodactyla. Repeats sites and short interspersed nucleotide elements (SINE) site, long interspersed nucleotide element (LINE) site are shown in figure

In a computer search for repeats sequences, two retrotransposons sequences short interspersed nucleotide elements (SINE) and long interspersed nucleotide elements (LINE) showed in intron1 region and exon3 region of porcine FSH $\beta$ gene (GenBank: D00621), respectively (Table 1). Zhao et al. (1999) found an extra SINE in intron 1 of porcine FSHB gene [7]. SINEs were considered to be genomic parasites and having no benefit to the organism (Batzer \& Deininger, 2002). The SINE insertion, found in introns of animal gene, may modify the gene's transcription pattern and its presence is well known to affect the expression level (Kramerov \& Vassetzky, 2005). The presence/absence of SINE resulted in allelic polymorphism of porcine FSH $\beta$ gene. The pig breeds with SINEallele was correlated with higher litter sizes than the breeds with SINE+ allele (Shi et al., 2006). When comparing with two SINE indicated that the SINE finding by zhao was located downstream of the SINE in FSH $\beta$ gene (GenBank: D00621) and their similarity are $80 \%$. The same high frequency SINE in pig due to probably positive selection during the evolutionary process of pig in artificial selection.

\section{Acknowledgments}

This research was funded by Science and Technology Agency of Sichuan province in China (2011JY0103).

\section{References}

Batzer, M., \& Deininger, P. L. (2002). Alu repeats and human genomic diversity. Nat. Rev. Genet, 3, 370-379. http://dx.doi.org/10.1038/nrg798 
Grigorova, M., Rull, K., \& Laan, M. (2007). Haplotype structure of FSHB, the beta-subunit gene for fertility-associated follicle-stimulating hormone: possible influence of balancing selection. Ann. Hum. Genet, 71, 18-28. http://dx.doi.org/10.1111/j.1469-1809.2006.00299.x

Kramerov, D. A., \& Vassetzky, N. S. (2005). Short retroposons in eukaryotic genomes. Int. Rev. Cytol, 247, 165-221. http://dx.doi.org/10.1016/S0074-7696(05)47004-7

Li, M. D., \& Ford, J. J. (1998). A comprehensive evolutionary analysis based on nucleotide and amino acid sequences of the alpha and beta subunits of glycoprotein hormone gene family. J. Endocrinol, 156, 529-542. http://dx.doi.org/10.1385/ENDO:11:3:205

McGee, E. A., \& Hsueh, A. J. (2000). Initial and cyclic recruitment of ovarian follicles. Endocr. Rev, 21, $200-214$. http://dx.doi.org/10.1210/er.21.2.200

Pierce, J. G., \& Parsons, T. F. (1981). Glycoprotein hormones: structure and function. Annu. Rev. Biochem, 50, 465-495. http://dx.doi.org/10.1146/annurev.bi.50.070181.002341

Plant, T.M., \& Marshall, G.R. (2001). The functional significance of FSH in spermatogenesis and the control of its secretion in male primates. Endocr. Rev, 22, 764-786. http://dx.doi.org/10.1210/er.22.6.764

Shi, Q. S., Liu X. C., Liu Z. W., Li X. P., Li Y. H., \& Tang, F. (2006). Effect of five genetic Loci related to pig litter size. Hereditas, 28, 652-658. Retrieved from http://europepmc.org/abstract/MED/16818425

Sun, L., Zhang, Z., Iqbal, J., Zaidi, S., Papachristou, D.J., Zhou, H., ... Zaidi, M. (2006). FSH directly regulates bone mass. Cell., 125, 247-260. http://dx.doi.org/10.1016/j.cell.2006.01.051

Ulloa-Aguirre, A., Timossi, C., amian-Matsumura P., \& Dias J. A. (1999). Role of glycosylation in function of follicle-stimulating hormone. Endocrine, 11, 205-215. http://dx.doi.org/10.1385/ENDO:11:3:205

Wallis, M. (2001). Episodic Evolution of Protein Hormones in Mammals. J. Mol. Evol, 53, 10-18. Retrieved from http://www.sciencedirect.com/science/article/pii/S0092867406003722

Zhao, Y. F., Li, N., Xiao, L., Cao, G. S., Chen. Y. Z., Zhang, S., .. Wu, C. X. (1998). FSHB subunit gene is associated with major gene controlling litter size in commercial pig breeds. Science in China, 41, 664-668. http://dx.doi.org/10.1007/BF02882910

Zhengwei, Y., Wreford, N. G., Schlatt, S., Weinbauer, G. F., Nieschlag, E., \& McLachlan, R. I. (1998). Acute and specific impairment of spermatogonial development by $\mathrm{GnRH}$ antagonist-induced gonadotrophin withdrawal in the adult macaque (Macaca fascicularis). J. Reprod. Fertil, 112, 139-147. http://dx.doi.org/10.1530/jrf.0.1120139 